DOI: http://dx.doi.org/10.12957/demetra.2014.11224

\title{
Especiarias e condimentos aumentam a ingestão alimentar de pacientes com dieta hipossódica?
}

\section{Do spices and condiments increase food intake of patients with low sodium diet?}

\author{
Maria Luiza Aires de Alencar ${ }^{1}$ \\ Anete Araúijo de Sousa' \\ Erasmo Benício Santos de Moraes Trindade 2 \\ 1 Programa de Pós-graduação em Nutrição, \\ Núcleo de Pesquisa de Nutrição em Produção \\ de Refeições. Universidade Federal de Santa \\ Catarina. Florianópolis-SC, Brasil. \\ 2 Programa de Pós-graduação em Nutrição. \\ Universidade Federal de Santa Catarina. \\ Florianópolis-SC, Brasil. \\ Correspondência /Correspondence \\ Anete Araújo de Sousa \\ Universidade Federal de Santa Catarina, \\ Departamento de Nutrição \\ Campus Universitário Trindade \\ 88040-900 - Florianopolis, SC - Brasil \\ E-mail:anete.sousa@ufsc.br
}

\section{Resumo}

Objetivo: o estudo avaliou se a ingestão alimentar de pacientes hospitalizados com prescrição de dieta hipossódica aumentou após a adição de especiarias e condimentos em preparações. Métodos: Ensaio clínico, não randomizado, controlado e cego realizado com pacientes de um hospital público de Florianópolis-SC. A amostra foi escolhida por conveniência. Os pacientes foram controles de si mesmos. Receberam em dois dias consecutivos almoço e jantar alternados da dieta hipossódica do padrão hospitalar e da dieta hipossódica modificada pela adição de especiarias e condimentos. A ingestão alimentar foi avaliada em gramas e percentual, comparando a "padrão" com a "modificada" e ambas, em relação às características dos pacientes, clínica de internação e dia de entrada na pesquisa. As análises foram efetuadas no software Stata 11.0. O nível de significância estatística estabelecido foi $\mathrm{p}<0,05$. Resultados: Concluíram o estudo 90 pacientes $(66 \%$ homens; média de 60 anos; $81 \%$ estudou de zero a oito anos; $56 \%$ não controlavam consumo de sal; $91 \%$ usavam medicamentos que alteram paladar; $81 \%$ internaram por doença crônica; $52 \%$ em clínica médica). Não houve diferença significativa de ingestão entre as dietas, em gramas $(p=0,794)$ e em percentual $(p=0,619)$, nem por sexo, idade, consumo de sódio/sal antes da admissão, clínica de internação, medicamentos envolvidos com distúrbio quimiosensório e dia de inserção na pesquisa. Houve diferença estatisticamente significativa quanto à escolaridade com maior aceitação da dieta "padrão" ( $<<0,001)$. Conclusão: A adição de especiarias e condimentos em preparações da dieta hipossódica não aumentou a ingestão alimentar de pacientes hospitalizados.

Palavras-chave: Dieta Hipossódica. Hipertensão. Especiarias. Ingestão de Alimentos. 


\section{Abstract}

Objective: To evaluate whether food intake of hospitalized patients on a prescribed low-sodium diet increased after addition of spices and condiments. Methods: Non-randomized, controlled and blind clinical test, performed with patients in a public hospital in the city of Florianopolis-SC, Brazil. The sample was chosen for convenience. Patients were their own control. They received on two consecutive days, alternated for lunch and dinner, the standard low-sodium hospital diet and modified low-sodium diet (with spices and condiments added). Food intake was evaluated in grams and percentages, comparing the "standard" with the "modified" diet and both in relation to the patient characteristics, care units and study start day. Analysis was carried out in Stata 11.0 software. The level of statistical significance was $\mathrm{p}<0.05$. Results: Ninety patients completed the study $(66 \%$ men; average aged 60 years; $81 \%$ with $0-8$ school years; $56 \%$ did not control salt intake; $91 \%$ used drugs which altered taste; $81 \%$ interned for chronic disease; $52 \%$ receiving general clinical care). There was no significant difference between the diets in terms of intake levels, in grams $(p=0.794)$ or in percentage $(p=0.619)$, nor according to gender, age, sodium/salt consumption before admission, care units, drugs involved with chemosensory disturbance and study start day. There was statistically significant difference regarding level of schooling with greater acceptance of the standard diet $(\mathrm{p}<0.001)$. Conclusion: The addition of spices and condiments to low-sodium diets did not increase the food intake of hospitalized patients.

Key words: Low-Sodium Diet. Hypertension. Spices. Food Intake.

\section{Introdução}

O sal é um alimento amplamente utilizado na alimentação humana, adicionado no preparo de refeições, em produtos industrializados e à mesa, devido a sua capacidade de melhorar aspectos sensoriais dos alimentos como textura, cor e sabor. ${ }^{1}$ Esta capacidade, associada ao comportamento descrito como "apetite humano por sal”, busca agradar ao paladar. ${ }^{2}$

Estudos têm associado o consumo elevado de sódio, proveniente principalmente do sal de cozinha, com hipertensão arterial sistêmica, doenças vasculares e cardíacas, insuficiência renal e hepática, câncer gástrico, osteoporose, asma e obesidade. ${ }^{3-5}$ Evidências científicas revelam, no entanto, que qualquer redução no consumo de sal diminui a pressão arterial ${ }^{3-5}$ e melhora o perfil de morbimortalidade por essas enfermidades. ${ }^{6}$ 
A Organização Mundial da Saúde $(\mathrm{OMS})^{4}$ recomenda uma ingestão menor que $5 \mathrm{~g}$ de sal por dia (equivalente a $2.000 \mathrm{mg} /$ dia de sódio) para pessoas saudáveis. Em doenças específicas, as restrições de sal e sódio são, por vezes, severas. Na insuficiência cardíaca, por exemplo, recomendase o consumo de até $2 \mathrm{~g}$ de sal/dia, equivalente a $800 \mathrm{mg}$ de sódio. ${ }^{7}$

A restrição do consumo de sal, embora necessária, é reconhecida pela baixa adesão dos pacientes,${ }^{8,9}$ decorrente principalmente dos hábitos alimentares e queixas de falta de sabor dos alimentos. ${ }^{10-13}$ A baixa adesão resulta em ingestão alimentar insuficiente e aporte de energia e nutrientes inferior às necessidades nutricionais. ${ }^{14,15}$

Modificações culinárias com o uso de especiarias, condimentos e técnicas de preparo têm sido recomendadas para encorajar o consumo de certos alimentos ou substituir parcial ou totalmente ingredientes que devem estar diminuídos ou ausentes nas dietas restritivas, como o sal na dieta hipossódica. ${ }^{16,17}$

Diante dos aspectos apontados, o objetivo do estudo foi avaliar se a ingestão alimentar de pacientes hospitalizados com prescrição de dieta hipossódica aumentava após a adição de especiarias e condimentos em suas preparações.

\section{Método}

\section{Participantes}

Estudo experimental, do tipo ensaio clínico não randomizado, controlado e cego, desenvolvido com pacientes internados em unidades de clínica médica, cirúrgica e emergência de um hospital público no estado de Santa Catarina, Brasil. Foram incluídos pacientes adultos, de ambos os sexos, com idade entre 25 e 85 anos e prescrição de dieta hipossódica de consistência branda ou normal, sem restrição de outros nutrientes, energia e/ou alimentos. A amostra foi definida por conveniência e saturação temporal. Para atender aos objetivos da pesquisa, cada paciente foi controle de si mesmo, motivo pelo qual a amostra não foi randomizada.

A pesquisa consistiu em comparar a ingestão alimentar de pacientes que receberam dieta hipossódica modificada por técnicas culinárias e adição de especiarias e condimentos, denominada "Dieta Hipossódica Modificada - DHM", com a dieta hipossódica padrão do hospital, denominada "Dieta Hipossódica Padrão - DHP”. A comparação foi realizada nas refeições de almoço e jantar em dois dias consecutivos, com pacientes internados entre o primeiro e o oitavo dia de admissão. A pesquisa ocorreu em dias úteis. O paciente foi inserido no estudo quando preenchia os critérios de inclusão e estava apto (sem jejum ou preparo para exames/cirurgias). 


\section{Modificações culinárias}

As modificações foram acrescentadas em três das quatro preparações quentes servidas no almoço: carnes, leguminosas e acompanhamentos. A escolha dos temperos e das técnicas culinárias foi baseada em recomendações da literatura. ${ }^{16,17}$ As quantidades foram definidas através de testespilotos. As modificações culinárias descritas abaixo foram realizadas em setor distinto da cozinha dietética, para não interferir na elaboração da dieta padrão:

Carnes: técnica da marinagem por 24 horas sob refrigeração na proporção de $1 \mathrm{Kg}$ de carne/1 porção de tempero (3 colheres de chá cheias de partes iguais de manjericão, alecrim e orégano secos, $100 \mathrm{ml}$ de água, $50 \mathrm{ml}$ de vinagre de álcool e $5 \mathrm{~g}$ alho fresco amassado). A DHP foi temperada antes da cocção com os ingredientes do setor.

- Leguminosas: modificadas após cocção na proporção de 1 porção de leguminosa (seis conchas)/1 porção de tempero (refogado fervente com $9 \mathrm{ml}$ de óleo composto de soja/oliva saborizado com alho e cebola, $2 \mathrm{~g}$ de alho fresco picado e $1 / 6$ de colher de chá padronizada de louro em pó).

- Acompanhamentos: modificados 5 minutos antes da finalização da cocção na proporção de 1 porção de acompanhamento (24 colheres de sopa cheias)/1 porção de tempero (3 colheres de chá cheias de ervas finas secas: salsa, cebolinha, estragão, cerefólio).

As marcas dos ingredientes foram padronizadas durante o experimento.

O cardápio do almoço da dieta branda hipossódica foi repetido no jantar dos dois dias de intervenção. No primeiro dia, o paciente recebia almoço DHM e jantar DHP. No segundo dia esta relação foi invertida, sendo o almoço DHP e o jantar DHM. O porcionamento de almoço e jantar foi realizado ao mesmo tempo para cada paciente. A refeição do jantar foi mantida sob refrigeração após o porcionamento e posteriormente reaquecida em forno combinado. As preparações foram produzidas sem adição de sal e ingredientes com sal. Todos os participantes receberam $1 \mathrm{~g}$ de sal e uma fatia de limão no almoço e jantar. Estas condutas faziam parte dos protocolos do setor.

\section{Coleta de dados e análise estatística}

Os dados sobre sexo, idade, escolaridade, consumo prévio de sódio/sal antes da admissão, razão de admissão e medicamentos prescritos (para avaliar aqueles envolvidos com distúrbio quimiosensório) e tipo de clínica de internação foram obtidos com o paciente ou em seu prontuário. 
As quantidades das refeições ofertadas e rejeitadas (em gramas) foram pesadas em balança digital da marca Filizola ${ }^{\circledR}$, modelo MF-30I (e=5g) para posterior cálculo da ingestão alimentar das dietas hipossódicas (DHP e DHM). O dia de inserção do paciente no experimento foi registrado para relacioná-lo à data de internação.

Os dados foram digitados com entrada dupla no programa Microsoft Office Excel 2003. A descrição da amostra foi expressa como frequência absoluta e relativa, apresentando características dos indivíduos, clínica de internação e dia de inserção no estudo. A estatística analítica foi usada para comparar a média de oferta, rejeição e ingestão de alimentos em gramas das DHP e DHM. Adicionalmente, foi estimada a ingestão percentual de cada indivíduo (ingestão em gramas / ofertado em gramas x 100). A média de ingestão das duas refeições foi verificada mediante a soma dos alimentos ingeridos no almoço e no jantar da dieta correspondente (DHP ou DHM), e posteriormente, dividido por dois. Para a descrição destas variáveis, foram usadas a média e o desvio padrão (DP) ou a mediana e o intervalo interquartil, segundo a simetria das variáveis. As possíveis associações foram posteriormente testadas com o teste $t$ ou o teste de Wilcoxon para dados pareados.

Para avaliar a associação da diferença média de ingestão entre a DHP e a DHM, conforme as variáveis relacionadas às características dos indivíduos e clínica de internação, foram usados os testes $t$ e o de Wilcoxon para dados não pareados no caso de variáveis dicotômicas, e o teste de ANOVA de heterogeneidade para variáveis politômicas.

O nível aceitável de significância estatística foi estabelecido como $\mathrm{p}<0,05$. Todas as análises foram realizadas através do software STATA 11.0 para Windows versão 11.0 (StatacorpLakeway Drive College Station, Texas, USA).

\section{Princípios éticos}

A pesquisa foi submetida ao Comitê de Ética em Pesquisa com Seres Humanos da Universidade Federal de Santa Catarina, aprovada sob o número 654, em 29 de março de 2010, e estava de acordo com os princípios éticos contidos na Declaração de Helsinki. Os participantes receberam orientação sobre os objetivos do estudo e, após lerem, assinaram o Termo de Consentimento Livre e Esclarecido (TCLE). 


\section{Resultados}

Dos 130 pacientes, 90 concluíram o estudo. A perda amostral ocorreu por prescrição de jejum nos dias de inserção no estudo (15), alta hospitalar (13), mudança nas rotinas do setor de produção de refeições com interferência nos protocolos da pesquisa (05), mudança para dieta diferente da branda ou normal hipossódica (03), desistência de pacientes (03) e apresentar náuseas durante a refeição (01).

A mediana do tempo de internação da amostra final foi de 14 dias (11,00 - 20,75 dias). Os pacientes foram registrados conforme a clínica em que iniciaram sua participação, sendo que $52 \%$ estavam em unidades de clínica médica.

As principais características dos participantes estão apresentadas na tabela 1 .

Tabela 1. Características dos participantes. Florianópolis-SC, 2010.

\section{Categorias}

Sexo

Masculino

Feminino

Faixa etária

$<60$ anos

$\geq 60$ anos

Escolaridade

0-8 anos

$>8$ anos

Controle do consumo de sal antes da internação

Não

Sim
$51(57 \%)$

\section{Frequência (\%)}

$59(66 \%)$

$31(34 \%)$

$45(50 \%)$

$45(50 \%)$

$73(81 \%)$

$17(19 \%)$

$39(43 \%)$ 


\section{Categorias}

Frequência $(\%)$

Clínica de internação

Médica

$47(52 \%)$

Cirúrgica

$31(35 \%)$

Emergência

Uso de medicação que altera o paladar e/ou olfato*

Não

Sim

Razões de Admissão

Doença cardíaca e/ou vascular

$34(38 \%)$

Outras doenças crônicas

$27(30 \%)$

Câncer

Doenças infecciosas

$9(10 \%)$

Diagnóstico a esclarecer

$8(9 \%)$

Dia de inserção na pesquisa em relação à data de internação

No 1ํ dia de internação

Do $2^{\circ}$ ao $4^{\circ}$ dia de internação

$45(50 \%)$

No $5^{\underline{0}}$ e $6^{\underline{0}}$ dias de internação

$27(30 \%)$

No 7o e 8o dias de internação

$11(12 \%)$

* Segundo tabela apresentada por Doty et al. ${ }^{24}$

Dois terços da amostra (66\%) foram do sexo masculino e $81 \%$ tinham apenas ensino fundamental. Entre os participantes, $57 \%$ referiram não restringir o consumo de sal antes da internação e $91 \%$ recebiam algum fármaco citado na literatura por causar distúrbios quimiossensórios.

Os medicamentos mais frequentes foram os anti-hipertensivos e medicações para doenças cardíacas, seguidos pelos agentes hipolipemiantes, os antibacterianos, antidepressivos e antivirais.

A análise da ingestão das duas dietas hipossódicas (DHP e DHM) encontra-se na tabela 2. 
Tabela 2. Diferenças de médias das quantidades ofertadas, rejeitadas e ingeridas (em gramas) nas diferentes refeições da DHM e da DHP. Florianópolis-SC, 2010.

\begin{tabular}{|c|c|c|c|c|c|c|c|}
\hline \multirow{2}{*}{ Refeição } & \multicolumn{2}{|r|}{ DHM } & \multicolumn{2}{|r|}{ DHP } & \multicolumn{3}{|c|}{ Diferença de médias } \\
\hline & M & DP & M & $\mathrm{DP}$ & M & IC95\% & $\mathrm{p}$ \\
\hline \multicolumn{8}{|l|}{ Almoço } \\
\hline Ofertada & 633,7 & 119,3 & 618,7 & 111,3 & 15,0 & $-20,0 ; 50,0$ & $0,398 *$ \\
\hline Rejeitada $\ddagger$ & 157,5 & $(65,0 ; 300,0)$ & 155,0 & $(60,0 ; 230,0)$ & 10,0 & $(-40,0 ; 95,0)$ & $0,188 \dagger$ \\
\hline Ingestão & 442,6 & 149,5 & 444,1 & 160,1 & $-1,5$ & $-35,2 ; 32,2$ & $0,930^{*}$ \\
\hline $\begin{array}{c}\text { Ingestão } \\
\text { percentual§ } \ddagger\end{array}$ & 73,8 & $(54,7 ; 89,0)$ & 76,6 & $(59,6 ; 88,2)$ & 0,0 & $(-11,1 ; 8,1)$ & $0,738 \dagger$ \\
\hline \multicolumn{8}{|l|}{ Jantar } \\
\hline Ofertada & 630,4 & 131,4 & 635,5 & 128,9 & $-5,1$ & $-40,1 ; 30,0$ & $0,775^{*}$ \\
\hline Rejeitada $\ddagger$ & 185,0 & $(70,0 ; 320,0)$ & 167,5 & $(85,0 ; 325,0)$ & 0,0 & $(-85,0 ; 80,0)$ & $0,867 \dagger$ \\
\hline Ingestão & 426,7 & 162,6 & 430,2 & 169,2 & $-3,4$ & $-37,6 ; 30,7$ & $0,842 *$ \\
\hline $\begin{array}{c}\text { Ingestão } \\
\text { Percentual§‡ }\end{array}$ & 71,5 & $(48,3 ; 88,7)$ & 72,1 & $(49,1 ; 86,5)$ & $-0,3$ & $(-12,2 ; 12,8)$ & $0,998 \dagger$ \\
\hline \multicolumn{8}{|l|}{$\begin{array}{l}\text { Média: } \\
\text { almoço/jantar }\end{array}$} \\
\hline Ofertada & 632,1 & 94,5 & 627,1 & 84,8 & 5,0 & $-15,2 ; 25,1$ & $0,627 *$ \\
\hline Rejeitada $\ddagger$ & 185,0 & $(82,5 ; 295,0)$ & 168,8 & $(92,5 ; 275,0)$ & 7,5 & $(-20,0 ; 35,0)$ & $0,198 \dagger$ \\
\hline Ingestão & 434,7 & 133,6 & 437,1 & 134,1 & $-2,5$ & $-21,1 ; 16,2$ & $0,794^{*}$ \\
\hline Ingestão & & & & & & & \\
\hline $\begin{array}{c}\text { Percentual } \\
\S \ddagger\end{array}$ & 69,5 & $(54,9 ; 86,4)$ & 74,6 & $(56,3 ; 84,6)$ & $-0,3$ & $(-4,4 ; 3,9)$ & $0,619 \dagger$ \\
\hline
\end{tabular}

\footnotetext{
* Teste t para dados pareados

$\uparrow$ Teste de Wilcoxon para dados pareados

† Os valores apresentados representam mediana e intervalo interquartil (entre parênteses)

$\S$ Relação percentual entre quantidade ingerida e ofertada
}

A ingestão de ambas as dietas foi similar, independentemente da refeição avaliada (almoço, jantar ou média de almoço e jantar), em gramas e em percentual. A ingestão das duas dietas ficou entre 70 (DHM) e 75\% (DHP), correspondendo à mediana de ingestão percentual das duas refeições. 
As diferenças de médias de ingestão entre DHM e DHP em relação às características dos participantes (tabela 3) mostraram que não houve diferença entre as duas dietas conforme sexo, faixa etária, controle do consumo de sal antes da internação, clínica de origem, uso de medicamentos que alteram o paladar e/ou olfato e dia de inserção na pesquisa. A análise em relação à escolaridade mostrou maior ingestão da DHP entre indivíduos que estudaram mais de oito anos $(\mathrm{p}<0,001)$.

Tabela 3. Diferenças de médias de ingestão ${ }^{\S}$ entre a DHM e a DHP, segundo características da população, clínica de internação e inserção no estudo. Florianópolis-SC, 2010.

Variáveis

Média da diferença de médias (IC95\%)

Valor $\mathrm{p}$

\section{Sexo}

Masculino

$3,6(-19,0 ; 26,3)$

$0,373^{*}$

Feminino

$-14,1(-48,5 ; 20,3)$

Faixa etária

$<60$ anos

$-4,9(-27,1 ; 17,2)$

$0,358 \dagger$

$\geq 60$ anos

$0,0(-31,0 ; 31,0)$

\section{Escolaridade}

0-8 anos

$13,4(-4,8 ; 31,7)$

$<0,001^{*}$

$>8$ anos

$-70,7(-123,6 ;-17,8)$

Controle do consumo de sal antes

\section{da internação}

Não

$8,7(-10,6 ; 28,1)$

$0,474 \dagger$

Sim

$-17,1(-52,7 ; 18,4)$

Clínica de internação

Médica

$-20,6(-45,2 ; 4,1)$

$0,084+$

Cirúrgica

$25,3(-4,1 ; 71,5)$

Emergência

$-3,1(-77,7 ; 71,5)$

Uso de medicação que altera o paladar

Não

$17,5(-45,3 ; 80,3) \quad 0,510 *$

Sim

$-4,4(-24,3 ; 15,5)$ 


\begin{tabular}{|c|c|c|}
\hline Variáveis & $\begin{array}{l}\text { Média da diferença de } \\
\text { médias (IC95\%) }\end{array}$ & Valor $\mathrm{p}$ \\
\hline \multicolumn{3}{|c|}{$\begin{array}{l}\text { Dia de inserção na pesquisa em relação à } \\
\text { data de internação }\end{array}$} \\
\hline No $1^{o}$ dia de internação & $57,5(-3,4 ; 118,4)$ & $0,09 \ddagger$ \\
\hline Do $2^{\mathrm{o}}$ ao $4^{\mathrm{o}}$ dia de internação & $-17,7(-47,3 ; 11,9)$ & \\
\hline No $5^{\underline{o}}$ e $6^{0}$ dias de internação & $-0,5(-30,7 ; 29,6)$ & \\
\hline No $7^{\circ}$ e $8^{0}$ dias de internação & $16,8(-37,2 ; 70,8)$ & \\
\hline
\end{tabular}

*Teste t para dados não pareados

$\dagger$ Teste de Wilcoxon para dados não pareados

†Teste de ANOVA de heterogeneidade

§Os valores apresentados representam a diferença média em gramas entre a quantidade ingerida na DHM (média do almoço e janta) e na DHP (média do almoço e janta). Valores positivos representam maior ingestão da DHM e valores negativos maior ingestão da DHP.

\section{Discussão e conclusão}

O estímulo sensorial decorrente das modificações culinárias sobre o aroma, sabor e apresentação das preparações hipossódicas não promoveu maior ingestão alimentar nos pacientes, revelando que outros fatores foram determinantes no resultado.

Yabuta et al., ${ }^{18}$ analisando a aceitação alimentar de pacientes que receberam dieta hipossódica em um hospital de cardiologia, observaram consumo de bom a ótimo entre 69,2\% dos pacientes. As preparações foram elaboradas sem sal e sem produtos industrializados com teor elevado de sódio. No entanto, a falta de sal foi referida como responsável pelo menor consumo alimentar $(21,1 \%)$ e pela insatisfação quanto ao sabor da refeição $(11,5 \%)$.

Heo et al., ${ }^{8}$ avaliando a adesão ao tratamento dietético com dieta hipossódica, observaram uma taxa de não adesão de 40\%. Neste estudo, alguns pacientes relataram ter consciência da necessidade de restrigir o sal, mas o prazer de comer foi considerado de maior valor, demonstrando que para eles a redução do sal implica perda do prazer de comer.

Demário et al..$^{10}$ observaram que a diminuição do apetite, o uso de medicamentos, o ambiente, a presença de acompanhante e aspectos sensoriais dos alimentos (apresentação, aparência e aroma), além do tipo de preparação, influenciaram na ingestão alimentar. A falta de sal e de tempero foi motivo de insatisfação, mesmo entre os pacientes com dieta normal. 
Os estudos citados indicam que a quantidade restrita de sal na dieta hipossódica interfere na percepção do paladar, com repercussões sobre a ingestão alimentar, satisfação quanto ao sabor das refeições e adesão ao tratamento. Destaca-se que, no presente estudo, a oferta de sal foi de 2g/dia (lg/almoço; $1 \mathrm{~g} /$ jantar), caracterizando redução severa em relação ao consumo habitual da população brasileira, estimado em $9,6 \mathrm{~g} / \mathrm{dia}^{19}$

Estudo sobre a aceitação de dietas com diferentes concentrações de sódio mostrou melhor aceitação para aquelas com médios teores $(2.300 \mathrm{mg})$, seguidas pelas de maiores $(3.500 \mathrm{mg}) \mathrm{e}$ finalmente pelas de menores concentrações $(1.200 \mathrm{mg})$. Os resultados indicaram que as dietas de baixo conteúdo de sódio foram tão bem aceitas quanto as outras, em um período de 30 dias de análises. Entretanto, as preparações utilizadas foram elaboradas em cozinha experimental com o sal embutido nos alimentos, seguindo o hábito culinário da população. ${ }^{20}$

O sal é conhecido por ser uma molécula sápida que estimula os receptores das papilas gustativas. Além disso, suprime seletivamente sabores desagradáveis e intensifica os agradáveis. ${ }^{21}$ No presente estudo, as preparações foram elaboradas sem sal, sendo adicionado sobre os alimentos no momento da refeição pelo próprio paciente. Este processo é comum nos hospitais. Porém, se diferencia do hábito das pessoas e pode influenciar a percepção do paladar, pois o sal se concentra na superfície dos alimentos e não se mistura em sua composição.

A escolaridade mostrou uma relação significativa com a ingestão alimentar dos pacientes estudados, sendo maior para a DHP ( $\mathrm{p}<0,001)$. A literatura consultada não discute esse tipo de relação. O número de indivíduos nesta categoria $(17=19 \%)$ pode ser um fator limitante para esta análise.

Quanto aos medicamentos envolvidos com distúrbios quimiossensórios, 91\% dos pacientes estudados recebiam um ou mais destes fármacos (tabela 1), o que possivelmente interferiu na ingestão de ambas as dietas (DHM e DHP). Entre os participantes, 13\% (tabela 1) faziam tratamento para doenças neoplásicas e possivelmente usavam quimioterápicos. Tais medicamentos, por estarem circulantes no sangue, são permeáveis à saliva e afetam os receptores do paladar, resultando no fenômeno de alteração intravascular do gosto que afeta o comportamento alimentar dos pacientes. ${ }^{22}$

Os anti-hipertensivos e medicações para cardíacos foram os mais prescritos entre os pacientes. Os anti-hipertensivos, quando mastigados ou mantidos na boca por longos períodos, produzem uma sensação de gosto amargo de longa duração, possivelmente ativado pelo sistema intravascular ou diretamente sobre os quimiorreceptores do paladar e do olfato. ${ }^{23} \mathrm{O}$ captopril, por exemplo, pode mudar o sabor doce de alimentos e deixar uma sensação de salgado, além de manter um sabor persistente de amargo ou salgado na boca, ${ }^{24}$ alterando a percepção do sabor dos alimentos. O uso dos medicamentos pode ter prejudicado a percepção de aroma e sabor pelos pacientes, tanto na DHM quanto na DHP. 
A suscetibilidade aos efeitos das drogas sobre o paladar depende de inúmeros fatores, incluindo sexo e idade. ${ }^{24}$ Os idosos são particularmente vulneráveis aos efeitos destes fármacos, ocorrendo redução da percepção do paladar e boca seca..$^{25}$ Destaca-se que $50 \%$ dos pacientes eram idosos (tabela 1). Desta forma, a ingestão alimentar pode ter sofrido influência de alterações gustativas próprias da idade, agravadas pelas condições de doenças ${ }^{26}$ e uso de medicamentos. ${ }^{24}$

As análises mostraram uma ingestão percentual de $70 \%$ e $75 \%$ para DHM e DHP, respectivamente (tabela 2). Esses valores coincidem com relatos da literatura sobre a ingestão alimentar de pacientes idosos hospitalizados. ${ }^{27}$

O presente estudo não teve como objetivo analisar se a quantidade ingerida foi capaz de atender às necessidades nutricionais dos pacientes envolvidos. No hospital em estudo, os pacientes receberam, além das refeições analisadas (almoço e jantar), três a quatro pequenas refeições (desjejum, lanches da manhã, da tarde e da noite). Portanto, a quantidade de alimentos ingerida pelos pacientes pode estar relacionada à saciedade, ${ }^{28}$ independentemente da adição ou não de modificações culinárias.

Thibault et al. ${ }^{29}$ analisaram a evolução do serviço de alimentação em um hospital em Genebra (Suíça) após dez anos de implementação de melhorias. A análise foi realizada em 1999 e em 2008, utilizando os mesmos procedimentos. Comparando-se os dois períodos, os resultados mostraram que $78 \%$ dos pacientes não ingeriam todos os alimentos nas principais refeições (desjejum, almoço e jantar). Em 2008, 25\% da refeição fornecida não foi consumida. Verificou-se que houve aumento de 15\% na prescrição de dietas restritivas. Mas, logo após a avaliação de 1999, a dieta hipossódica foi suprimida do hospital.

Outro aspecto a ser considerado relaciona-se com a baixa expectativa e a experiência com a comida de hospital, que contrasta desfavoravelmente com a comida de casa. ${ }^{30}$ Dallepiane \& Bós, ${ }^{12}$ introduzindo a utilização de condimentos sem sal entre pacientes ambulatoriais, obtiveram resultados positivos na adesão ao tratamento dietético e na palatabilidade da alimentação. No entanto, a intervenção e as refeições foram realizadas no domicílio dos pacientes, o que pode revelar a influência do ambiente hospitalar sobre a ingestão de dietas modificadas.

A necessidade de padronização das modificações culinárias do experimento impôs um mesmo sabor para todos os pacientes. No entanto, os hábitos alimentares são plurais ${ }^{31}$ e, portanto, os condimentos preferidos por alguns podem não ser apreciados por outros. As ervas finas utilizadas nas preparações de acompanhamento e a marinagem para as carnes são pouco usadas na culinária local. O louro, por sua vez, é comumente usado em folha, e não em pó, produzindo um sabor mais suave. Possivelmente, com a utilização de condimentos incorporados ao hábito alimentar dos pacientes, os resultados fossem diferentes, pois os indivíduos e os grupos aceitam melhor aquilo que já conhecem, incluindo as regras culinárias e seus significados. ${ }^{32}$ 
Concluindo, a adição de especiarias, condimentos e técnicas culinárias em preparações da dieta hipossódica não aumentou a ingestão alimentar dos pacientes estudados. No entanto, os resultados apontam uma possível influência na ingestão, relacionada ao reduzido teor de sal ofertado na dieta e adição de sal nas preparações prontas. Estes fatores podem ter sido determinantes, independentemente da adição de especiarias e condimentos, dos tipos de temperos utilizados e do uso de medicamentos. Os aspectos abordados podem gerar novas hipóteses e ser objeto de novos estudos de intervenção.

\section{Agradecimentos}

Aos pacientes e funcionários do hospital que possibilitaram a realização da pesquisa.

\section{Referências}

1. UK. Trading Standards Institute. The salt reduction toolkit. United Kingdom: Trading Standards Institute, 2008. [citado 2010 Jan 27]. Disponível em: http://www.tradingstandards.gov.uk/policy/ policy-saltreductiontoolkit.cfm

2. Leshem M. Biobehavior of the human love of salt. Neurosci and Biobehav Rev. 2009; 33: 1(17). doi:10.1016/j.neubiorev.2008.07.007

3. He FJ, Macgregor GA. Reducing population salt intake worldwide: from evidence to implementation. Prog Cardiovasc Dis. 2010; 52:363-382. doi:10.1016/j.pcad.2009.12.006

4. World Health Organization - WHO. Report of a WHO forum and technical meeting: Reducing Salt Intake in Populations. Paris, France, 5-7 Oct. 2006. Paris, France, 2007.

5. Intersalt Cooperative Research Group. Intersalt: an international study of electrolyte excretion and blood pressure. Results for 24 hour urinary sodium and potassium excretion. BMJ 1988; 297: 319-328. doi:10.1136/bmj.297.6644.319

6. Public Health Agency of Canada-PHAC. Dropping the salt. Pratical step countries are taking to prevent chronic non-communicable diseases through population-wide dietary salt reduction. Pan American Health Organization and World Health Organization, 2009. [citado 2010 Abril 25]. Disponível em: http://www.paho.org/English/AD/dpc/nc/salt-mtg-phac-paper.pdf

7. Nakasato M, Strunk CMC, Guimarães G, Rezende MVC, Bocchi EA. A dieta com Baixo Teor de Sódio é de Fato indicada para todos os Pacientes com Insuficiência Cardíaca Estável? Arq Bras Cardiol. 2010; 94(1):92-101.

8. Heo S, Lennie TA, Moser DK, Okoli C. Heart failure patients' perceptions on nutrition and dietary adherence. Eur J Cardiovasc Nurs. 2009; 8:323-328. doi:10.1016/j.ejcnurse.2009.05.005 
9. Ahmed N, khaliq MA, Shah SH, Anwar W. Compliance to antihypertensive drugs, salt restriction, exercise and control of systemic hypertension in hypertensive patients at abbottabad. J Ayub Med Coll Abbottabad. 2008; 20:66-69.

10. Demário RL, Sousa AA, Salles RK. Comida de hospital: percepções de pacientes em um hospital público com proposta de atendimento humanizado. Ciênc. Saúde Colet. 2010; 15(Supl. 1):1275-1282.

11. Coloço RB, Holanda LB, Portero-Mclellan KC. Determinantes do grau de satisfação de pacientes internados referente a refeições oferecidas em um hospital universitário. Rev Ciênc Méd. 2009; 18:121130.

12. Dallepiane LB, Bós AJG. O uso de condimentos na dieta em um grupo de hipertensos: estudo de intervenção randomizado. Rev Bras Nutr Clin. 2007; 22:286-9.

13. Sacks FM, Campos H. Dietary Therapy in Hypertension. N. Engl. J. Med. 2010; 362:2102-2112.

14. Kyungjoo K, Minyoung K, Kyung-Eun L. Assessment of foodservice quality and identification of improvement strategies using hospital foodservice quality model. NRP 2010; 4:163-172. doi: 10.4162/ nrp.2010.4.2.163

15. Dupertuis YM, kossovsky MP, kyle UG, Raguso C A, Genton L, Pichard C. Food intake in 1707 hospitalized patients: a prospective comprehensive hospital survey. Clin Nutr. 2003; 22:115-123. doi:10.1054/clnu.2002.0623

16. Spano M. Cooking With Herbs and Spices More Flavor, Better Health. Diabetes Self Manag 2009 january 20. [citado 2011 Feb 18]. Disponível em: http://www.diabetesselfmanagement.com/articles/ nutrition-and-meal-planning/cooking_with_herbs_and_spices/all/).

17. Fazio VA, Inge KE. Health benefits of herbs and spices: the past, the present, the future - Dietary implications. Med J Aust. 2006; 21;185(4 Suppl):S4-24.

18. Yabuta CY, Cardoso E, Isosaki M. Dieta hipossódica: aceitação por pacientes internados em hospital especializado em cardiologia. Rev Bras Nutr Clin. 2006; 21:33-7.

19. Ministério da Saúde (Brasil), Secretaria de Atenção à saúde, Coordenação-Geral da Política de Alimentação e Nutrição. Guia alimentar para a população brasileira: promovendo alimentação saudável. Brasília: Ministério da Saúde, 2008.

20. Karanja N, Lancaster KJ, Vollmer WM, Lin P-H, Most MM, Ard JD, et al. Acceptability of sodiumreduced research diets, including the dietary approaches to top hypertension diet, among adults with prehypertension and stage 1 hypertension. J Am Diet Assoc. 2007; 107:1530-1538. doi: 10.1016/j. jada.2007.06.013

21. Hervé T. Como o sal modifica o gosto: o sabor salgado transforma e atenua os sabores amargos e doces. [Edições especiais] A ciência na cozinha: Corpo, máquina de comer. Scientific American Brasil; 2007: ed. 02; p. 40-41. [citado 2011 nov 11]. Disponível em: http://www2.uol.com.br/sciam/ edicoes_anteriores/_10.html

22. Sánchez-Lara K, Sosa-Sánchez R, Green-Renner D, Rodríguez C, Laviano A, Motola-Kuba D, et al. Influence of taste disorders on dietary behaviors in cancer patients under chemotherapy. Nutrition Journal 2010; 9:15. doi:10.1186/1475-2891-9-15 
23. Doty RL, Philip S, Reddy K, Kerr KL. Influences of antihypertensive and antihyperlipidemic drugs on the senses of taste and smell: a review. JHypertension. 2003; 21:1805-1813. doi: 10.1097/01. hjh.0000084769.37215.16

24. Doty RL, Shah M, Bromley SM. Drug-induced taste disorders. Drug Saf. 2008; 31:199-215.

25. Shinkai RS, Hatch JP, Schmidt CB, Sartori EA. Exposure to the oral side effects of medication in a community-based sample. Spec Care Dentist. 2006; 26:116-20. doi: 10.1111/j.1754-4505.2006. tb01434.x

26. Watanabe M, Kudo H, Fukuoka Y, Hatakeyama A, Kudo H, Kodama H, et al. Salt taste perception and salt intake in older people. Geriatr Gerontol Int. 2008; 8:62-64. doi: 10.1111/j.1447-0594.2008.00448.x

27. Walton K, Williams P, Tapsell L, Batterham M. Rehabilitation inpatients are not meeting their energy and protein needs. e-SPEN Journal 2007; 2:120-126. doi:10.1016/j.eclnm.2007.09.001

28. Willem F, Nieuwenhuizen WF, Weenen H, Rigby P, Hetherington MM. Older adults and patients in need of nutritional support: Review of current treatment options and factors influencing nutritional intake. Clinical Nutrition 2010; 29(2):160-169. doi:10.1016/j.clnu.2009.09.003

29. Thibault R, Chikhi M, Clerc A, Darmon P, Chopard P, Genton L, et al. Assessment of food intake in hospitalised patients: A 10-year comparative study of a prospective hospital survey. Clinical Nutrition. Clin Nutr. 2011; 30(3):289-96. Epub 2010 Nov 9, doi:10.1016/j.clnu.2010.10.002.

30. Johns N, Hartwell H, Morgan M. Improving the provision of meals in hospital. The patients' viewpoint. Appetite 2010; 54:181-185. doi:10.1016/j.appet.2009.10.005

31. Corbeau J-P. Alimentar-se no Hospital: as dimensões ocultas da comensalidade. In: Canesqui A M, Garcia RWD. Antropologia e nutrição: um diálogo possível. Rio de Janeiro: Fiocruz; 2005: 9-19. (Coleção antropologia e saúde).

32. Sydner YM, Fjellström C. The meaning of symbols of culinary rules - the food and meals in elderly care. J Foodserv. 2006; 17:182-8. doi: 10.1111/j.1745-4506.2006.00036.x 
